\title{
HOMOTOPY RIGIDITY FOR GRASSMANNIANS
}

\author{
ALLEN BACK ${ }^{1}$
}

\begin{abstract}
Two $n$-dimensional unitary representations which differ by complex conjugation or tensoring with a character induce topologically equivalent actions on the Grassmann manifold of complex $m$-planes in $n$-space. This paper shows under modest dimension hypotheses that only such projectively equivalent linear representations of compact connected Lie groups can give topologically conjugate actions.
\end{abstract}

A linear representation $\alpha: G \rightarrow U(N)$ induces a $G$-action on the Grassmann manifold $G_{m}\left(C^{N}\right)=U(N) / U(m) \times U(N-m)$ of $m$-planes in complex $N$-space. If $\bar{\alpha}$ is the conjugate of $\alpha$ and $\chi$ is any character of $G$, it is obvious that $\alpha \otimes \chi$ and $\bar{\alpha} \otimes \chi$ give rise to the same actions. The purpose of this paper is to show for most values of $m$ and $N$ that only such obviously equivalent linear actions give topologically conjugate actions on $G_{m}\left(C^{N}\right)$. The main result is as follows.

TheOREM 1. Let $\alpha$ and $\beta$ be $N$-dimensional unitary representations of a compact connected Lie group $G$. Suppose that there is a homotopy equivalence $f: G_{m}\left(C^{N}\right) \rightarrow$ $G_{m}\left(C^{N}\right)(N>m)$ which is a $G$-map between $G_{m}\left(C^{N}\right)$ with the two linear actions $\alpha$ and $\beta$. Further suppose that either

(a) $N \neq 2 m$, or

(b) $m<3$.

Then there is a character $\chi: G \rightarrow S^{1}$ so that $\beta$ is equivalent as a linear representation to either $\alpha \otimes \chi$ or $\bar{\alpha} \otimes \chi$.

In the language of Arunas Liulevicius ([1], [2], [3], [4]), this theorem says that compact connected Lie group actions on $G_{m}\left(C^{N}\right)$ satisfy the property of homotopy rigidity. Wu-Yi Hsiang observed that such results could sometimes be proved efficiently by the use of equivariant cohomology (see e.g. [5]). We shall use this technique to prove Theorem 1. The author would like to express his appreciation to Wu-Yi Hsiang, Arunas Liulevicius, and Birgit Speh for helpful comments.

Because equivariant cohomology is invariant under $G$-maps which are homotopy equivalences, our strategy for proving Theorem 1 is quite straightforward. We shall simply show that two linear actions which are not obviously equivalent will in fact have nonisomorphic equivariant cohomologies.

The dimension restrictions in Theorem 1 arise completely from uncertainty about the group of automorphisms of the integral cohomology of the Grassman-

Received by the editors May 21, 1979 and, in revised form, September 29, 1979.

AMS (MOS) subject classifications (1970). Primary 57E25; Secondary 57E15, 57F15.

${ }^{1}$ Work supported in part by NSF Grant MCS 77-01623. 
nian in this case. It is a very viable conjecture that only the obvious automorphisms occur.

In order to study the equivariant cohomology, we shall recall some results about the cohomology of the Grassmannian (see e.g. [6]). As is well known, the cohomology of the infinite Grassmannian of $m$-planes may be identified with the algebra of symmetric functions in $m$-variables $t_{1}, t_{2}, \ldots, t_{m}$. The elementary symmetric functions $\sigma_{1}, \sigma_{2}, \ldots, \sigma_{m}$ are then the Chern classes of the canonical $m$-plane bundle. Define classes $h_{k} \in H^{2 k}(B U(m) ; Z) \subseteq Z\left[t_{1}, \ldots, t_{m}\right]$ inductively by

$$
\begin{aligned}
h_{0} & =1 \quad\left(h_{i}=0 \text { for } i<0\right), \\
h_{n+m} & =\sum_{j=1}^{m}(-1)^{j-1} \sigma_{j} h_{n+m-j} .
\end{aligned}
$$

Let $\left\langle h_{N-m+1}, h_{N-m+2}, \ldots, h_{N}\right\rangle$ be the ideal in $Z\left[h_{1}, \ldots, h_{m}\right]=Z\left[\sigma_{1}, \ldots, \sigma_{m}\right]$ generated by $h_{N-m+1}, \ldots, h_{N}$.

THEOREM 2. (1) $H^{*}\left(G_{m}\left(C^{N}\right) ; Z\right)=Z\left[h_{1}, \ldots, h_{m}\right] /\left\langle h_{N-m+1}, \ldots, h_{N}\right\rangle$.

(2) $A$ module basis for $H^{*}\left(G_{m}\left(C^{N}\right)\right.$; $\left.Z\right)$ is given by all $m$-fold products

$$
h_{i_{1}} h_{i_{2}} \cdots h_{i_{m}} \text { with } 0 \leqslant i_{1} \leqslant i_{2} \leqslant \cdots<i_{m}<N-m \text {. }
$$

The $h_{k}$ are the so-called "complete symmetric functions." They may be identified (up to sign) with the Chern classes of the canonical $N-m$-plane bundle. Their generating function $H=\sum_{k=0}^{\infty} h_{k}$ is given by

$$
\prod_{j=1}^{m}\left(\frac{1}{1-t_{j}}\right)=1 /\left(\sum_{j=0}^{m}(-1)^{j} \sigma_{j}\right) \text {, }
$$

and hence $h_{k}$ can also be described as the unique degree $k$ symmetric polynomial each of whose monomials $t_{1}^{i_{1}} t_{2}^{i_{2}} \cdots t_{m}^{i_{m}}$ has coefficient 1 .

For compact connected Lie groups, similarity of linear respresentations is entirely detected by restriction to a maximal torus. Also any character of the maximal torus which is invariant under the Weyl group action will be the restriction of a character of the group. Hence it suffices to prove Theorem 1 when $G$ is a torus $T$. Let $R=H^{*}(B T ; Z)=Z\left[x_{1}, \ldots, x_{p}\right]$ where $p$ is the rank of the torus. Note that by transgression, the weights of a representation $\alpha: T \rightarrow U(N)$ may be viewed as two dimensional elements of $R$.

Recall that the equivariant cohomology $H_{T}^{*}(X)$ of a $T$-space $X$ is defined to be the ordinary cohomology of the space $X_{T}$ formed by the Borel construction. More precisely, $X_{T}$ is the total space of the bundle

$$
\begin{gathered}
X \rightarrow X_{T}=E T \times{ }_{T} X \\
\downarrow \\
\quad B T
\end{gathered}
$$

which is associated to the universal $T$-bundle by the $T$-action on $X$.

Now in the case where $X=G_{m}\left(C^{N}\right)$ with a linear $T$-action, the canonical bundles $\gamma^{m}$ and $\gamma^{N-m}$ are equivariant bundles. Hence the Borel construction may be applied to them to give bundles $\left(\gamma^{m}\right)_{T}$ and $\left(\gamma^{N-m}\right)_{T}$ over $X_{T}$. Inasmuch as the 
Chern classes $c_{i}\left(\gamma^{m}\right)=\sigma_{i}$ generate $H^{*}(X ; Z)$, we see that the Chern classes of $\left(\gamma^{m}\right)_{T}$ provide explicit lifts of $H^{*}(X ; Z)$ to $H_{T}^{*}(X ; Z)$. Hence the fiber of the Borel fibration is totally nonhomologous to zero. Also for the trivial bundle of dimension $N$ with nontrivial torus action given by $\alpha$, it is easy to see that $c_{k}\left(\xi_{T}\right)$ is the $k$ th elementary symmetric function $\rho_{k}$ of the weights $w_{i}$ of $\alpha$. As $\left(\gamma^{m}\right)_{T} \oplus\left(\gamma^{N-m}\right)_{T}=$ $\xi_{T}$, we may use the above observations to obtain the following (see [6] for more detail).

THEOREM 3. Let $T$ act on $G_{m}\left(C^{N}\right)$ by a linear representation $\alpha$ with weights $w_{i}$. Then

$$
H_{T}^{*}\left(G_{m}\left(C^{N}\right) ; Z\right)=R\left[h_{1}, \ldots, h_{m}\right] /\left\langle f_{N-m+1}, \ldots, f_{N}\right\rangle
$$

where

$$
f_{m}=\sum_{j=0}^{M}(-1)^{j} \rho_{j} h_{m-j}
$$

and $\rho_{j}$ is the jth elementary symmetric function of the weights $w_{i}$.

It is worth noticing that the classes $h_{k} \in H_{T}^{*}\left(G_{m}\left(C^{N}\right) ; Z\right)$ are defined by the recursion formulas (*) where the $\sigma_{i}$ are now taken to be the Chern classes $c_{i}\left(\left(\gamma^{m}\right)_{T}\right)$. The bundle $\gamma^{m}$ with action $\alpha \otimes \chi$ is $T$-isomorphic to the tensor product of $\gamma^{m}$ with action $\alpha$ and a 1-dimensional trivial bundle with action $\chi$. Let $w$ be the weight of $\chi$. Then replacement of $\alpha$ by $\alpha \otimes \chi$ replaces the elementary symmetric function $\sigma_{k}$ of $t_{1}, \ldots, t_{m}$ by the corresponding symmetric function of $t_{1}+w, \ldots, t_{m}+w$.

By restriction to the fiber in the Borel bundle, it is clear that any isomorphism of equivariant cohomologies which is induced by a $T$-map will in turn induce an automorphism of $H^{*}\left(G_{m}\left(C^{N}\right) ; Z\right)$. The following has been shown by Brewster [9] (see also [8] and [7] for special cases with simpler proofs).

TheORem 4. Suppose $N \geqslant 3$. If $N \neq 2 m$, the only nontrivial automorphism of $H^{*}\left(G_{m}\left(C^{N}\right) ; Z\right)$ is the involution induced by conjugation $c$ of complex numbers. Explicitly $c^{*}\left(h_{k}\right)=(-1)^{k} h_{k}$.

The above result is a special case of [2, Conjecture 5].

CONJECTURE. If $N=2 m, m>1$ then the group of automorphisms is $Z / 2 \times$ $Z / 2$ generated by conjugation and the map $d$ taking an $m$-plane to the $m$-plane orthogonal to it. Explicitly $d^{*}\left(h_{m}\right)=(-1)^{m} \sigma_{m}$. The cases $(N, m)=(4,2)$ or $(6,3)$ are easy to verify. Consequently:

LEMMA 5. With the dimension restriction of Theorem 1, all automorphisms of $H^{*}\left(G_{m}\left(C^{N}\right) ; Z\right)$ are induced by maps which are equivariant between $G_{m}\left(C^{N}\right)$ with action $\alpha$ and $G_{m}\left(C^{N}\right)$ with action either $\alpha$ or $\bar{\alpha}$.

In proving Theorem 1, we will also establish homotopy rigidity for any value of $N=2 m$ where Lemma 5 remains valid.

It is also helpful to define a filtration of $R\left[h_{1}, \ldots, h_{m}\right]$. Let $J_{k}$ be the ideal 
generated by the elements of $R$ having degree greater than or equal to $k$. (Here the degree of an element is one half its dimension as a cohomology class; i.e. $\operatorname{deg} x_{i}=1$.) Clearly $J_{r} \cdot J_{s}=J_{r+s}$ and $\cap_{k=0}^{\infty} J_{k}=\{0\}$. Also define $S$ to be the linear span (over $R$ ) of all singletons $h_{k}$. Notice that the generators of the ideal of relations in $H_{T}^{*}$ are elements of $S$. The following is the essential technical lemma required to prove Theorem 1 . We will use the convention $h_{x}=0$ if $x<0$.

Lemma 6. Suppose $\Phi$ is a graded endomorphism of the algebra $R\left[h_{1}, \ldots, h_{m}\right]$ satisfying

$$
\Phi\left(\sigma_{i}\right)=\sigma_{i}+A_{i} \text { modulo } J_{k+1} \quad(k>1)
$$

where $A_{i} \in J_{k}$ for $1<i<m$.

Define elements $\boldsymbol{P}_{r, j}$ by

$$
P_{r, j}=\left[\sum_{i=1}^{m}(-1)^{i-1} A_{i} h_{j-i}\right] h_{r-j}
$$

Then

$$
\Phi\left(h_{r}\right)=h_{r}+\sum_{j=1}^{r} P_{r, j} \quad \text { modulo } J_{k+1} .
$$

Proof. The proof is by induction on $r$. The cases $r<1$ are trivial. Assume the truth of the lemma for $r<s$. Since $h_{s}=\sum_{i=1}^{m}(-1)^{i-1} \sigma_{i} h_{s-i}$, we note that the $P_{s, j}$ satisfy the recurrence relation

$$
P_{s, j}=\sum_{i=1}^{m}(-1)^{i-1} \sigma_{i} P_{s-i, j} \text { for } s>j
$$

with the convention $P_{s, j}=0$ if $s<j$. Now

$$
\begin{aligned}
\Phi\left(h_{s}\right)= & \sum_{i=1}^{m}(-1)^{i-1} \Phi\left(\sigma_{i}\right) \Phi\left(h_{s-i}\right) \\
= & \sum_{i=1}^{m}(-1)^{i-1}\left(\sigma_{i}+A_{i}\right)\left(h_{s-i}+\sum_{j=1}^{s-i} P_{s-i, j}\right) \text { modulo } J_{k+1} \\
= & \sum_{i=1}^{m}(-1)^{i-1} \sigma_{i} h_{s-i}+\sum_{i=1}^{m}(-1)^{i-1} A_{i} h_{s-i} \\
& +\sum_{i=1}^{m} \sum_{j=1}^{s-i}(-1)^{i-1} \sigma_{i} P_{s-i, j} \text { modulo } J_{k+1} .
\end{aligned}
$$

But

$$
\sum_{i=1}^{m} \sum_{j=1}^{s-i}(-1)^{i-1} \sigma_{i} P_{s-i, j}=\sum_{j=1}^{s-1} \sum_{i=1}^{m}(-1)^{i-1} \sigma_{i} P_{s-i j}=\sum_{j=1}^{s-1} P_{s, j} .
$$

Thus $\Phi\left(h_{s}\right)=h_{s}+\sum_{j=1}^{s} P_{s, j}$.

One of the complications of using Theorem 2 is that the product of two basis elements of $H^{*}\left(G_{m}\left(C^{N}\right) ; Z\right)$ cannot in general be readily expanded in terms of the basis. Fortunately, if we express $A_{i}$ in terms of this basis, then the expressions $\boldsymbol{P}_{r, j}$ in Lemma 6 will contain at most $m+1$-fold products of the $h_{n}$ 's. An $m+1$-fold 
product will occur only when $k=1$ and $A_{m}$ has a term $w h_{1}^{m-1}$ in its expansion. In all other cases, the monomials in $P_{r, j}$ will already be basis elements.

To avoid computation of $h_{1}^{m-1} h_{j-m} h_{r-j}$, Lemma 6 will be used only in the following case.

LEMMA 7. In addition to the assumptions of Lemma 6, assume $A_{m}$ has no $w h_{1}^{m-1}$ term in its expansion. (This is automatic if $k>1$.) Then if $N>2 m$ and $m>2$,

$$
\Phi\left(h_{N-m+1}\right) \in S \text { (the singletons) modulo } J_{k+1}
$$

if and only if $A_{i} \in J_{k+1}$ for all $i$.

Proof. By Lemma 6, $\Phi\left(h_{N-m+1}\right) \in S$ iff

$$
\sum_{i=1}^{m}(-1)^{i-1} A_{i} \sum_{j=1}^{N-m+1} h_{j-i} h_{N-m+1-j} \text { modulo } J_{k+1}
$$

lies in $S$. Since $A_{i} \in J_{k}, A_{i}=0$ for $i<k$. Upon expressing $A_{i}$ relative to the basis of Theorem 2, noting that all products are less than or equal to $m$-fold, and observing $\sum_{j=1}^{N-m+1} h_{j-i} h_{N-m+1-j}=2 h_{N-m+1-i}+$ doubletons, it now follows by induction on $i$ that $A_{i} \in J_{k+1}$ for all $i$.

Proof of Theorem 1. We shall assume $N \geqslant 2 m$ and $m \geqslant 2$. (The case $m=1$ is complex projective space where the technique of this paper works almost trivially upon factoring the relation $f_{N}^{\alpha}$ into linear factors.)

By Theorem 3, the isomorphism $f^{*}$ on equivariant cohomology is induced by an endomorphism $\Phi$ of $R\left[h_{1}, \ldots, h_{m}\right]$. Because the relations in $H_{T}^{*}$ occur in dimensions above those of the generators, $\Phi$ is uniquely determined by $f^{*}$. Modulo $J_{1}, \Phi$ must give one of the automorphisms of $Z\left[h_{1}, \ldots, h_{m}\right]$ described in Lemma 5 . As each is induced by an equivariant map, by composing with one of these, we may assume

$$
\Phi\left(\sigma_{i}\right)=\sigma_{i}+A_{i}
$$

where $A_{i} \in J_{1}$ for $1 \leqslant i \leqslant m$.

Suppose $A_{m}$ has a term $w h_{1}^{m-1}$ in its expansion where $w \in R$. Let $\chi$ be the character with weight $-w$. Then replacing $\alpha$ by $\alpha \otimes \chi$ replaces $\sigma_{m}$ by the $m$ th elementary symmetric function of $t_{1}-w, t_{2}-w, \ldots, t_{m}-w$. Modulo $J_{1}$, this is just $\sigma_{m}-w \sigma_{m-1}$. However it follows readily by induction that the expansion of $\sigma_{m-1}$ in terms of the basis of Theorem 2 contains $h_{1}^{m-1}$ with coefficient 1 . Thus with the replacement of $\alpha$ by $\alpha \otimes \chi$, we may assume that $A_{m}$ has no $w h_{1}^{m-1}$ terms in it.

Now $\Phi$ must take the ideal $\left\langle f_{N-m+1}^{\alpha}, \ldots, f_{N}^{\alpha}\right\rangle$ into the ideal $\left\langle f_{N-m+1}^{\beta}, \ldots, f_{N}^{\beta}\right\rangle$. In particular, $\Phi\left(f_{N-m+1}^{\alpha}\right)=f_{N-m+1}^{\beta}$. Notice that both $f_{N-m+1}^{\alpha}$ and $f_{N-m+1}^{\beta}$ belong to $S$. But modulo $S$ and $J_{2}, \Phi\left(f_{N-m+1}^{\alpha}\right)=\Phi\left(h_{N-m+1}\right)$. Hence by Lemma 7, $A_{i} \in$ $J_{2}$. Continuing shows $A_{i} \in J_{k}$ for all $k$; i.e. $A_{i}=0$ for all $i$. Thus $\Phi$ is the identity.

As $\Phi$ preserves the ideal of relations, $\Phi\left(f_{N}^{\alpha}\right)=\sum_{i=0}^{m-1} u_{i} f_{N-i}^{\beta}$. Consideration of this ideal modulo $J_{1}$ shows $u_{0}=1$ and $u_{i} \in J_{1}$ for $i>0$. Inductively considering this relation modulo $J_{k}$ for $k=1,2,3, \ldots, m$ shows in fact that $u_{i}=0$ for $i>0$. Thus $\Phi\left(f_{N}^{\alpha}\right)=f_{N}^{\beta}$. But the coefficients of $h_{k}$ in these relations are up to sign just the 
$N-k$ th symmetric functions of the weights of the two representations. Accordingly, all symmetric functions of the weights of $\alpha$ and $\beta$ must agree. Since the weights themselves are just the roots of the polynomial $h(t)=\sum_{j=0}^{N}(-1)^{j} \rho_{j} t^{N-j}$, it is immediate that $\alpha$ and $\beta$ have the same set of weights; i.e. that they are equivalent as linear representations.

Notice with trivial changes in dimensions that the analogous result for symplectic linear actions on quaternionic Grassmann manifolds also follows.

\section{BIBLIOGRAPHY}

1. Arunas Liulevicius, Characters do not lie, Transformation Groups (Ed., Czes Kosniowski), Proc. Conf. on Transformation Groups (Newcastle upon Tyne, August 1976), Cambridge University Press, London and New York, 1976, pp. 139-146.

2. __ Homotopy rigidity of linear actions: characters tell all, Bull. Amer. Math. Soc. 84 (1977), 213-221.

3. __ Flag manifolds and homotopy rigidity of linear actions, Proc. Canad. Conf. at the Univ. of British Columbia, August 1977.

4. Arunas Liulevicius and John Ewing, Homotopy rigidity of linear actions on friendly homogeneous spaces (to appear).

5. Wu-Yi Hsiang, Cohomology theory of topological transformation groups, Springer-Verlag, New York, 1975.

6. Allen Back, Involutions of Grassmann manifolds, Thesis, Univ. of California, Berkeley, 1977.

7. L. O'Neill, The fixed point property for Grassmann manifolds, Ph.D. Dissertation, Ohio State Univ., 1974.

8. Henry Glover and Bill Homer, Endomorphisms of the cohomology ring of finite Grassmann manifolds, Proc. Northwestern Univ. Homotopy Theory Conf., March 1977.

9. Stephen Brewster, Automorphisms of the cohomology ring of finite Grassmann manifolds, Dissertation, Ohio State Univ., 1978.

Department of Mathematics, The University of Chicago, Chicago, Ilunnois 60637

Current address: Department of Mathematics, Cornell University, Ithaca, New York 14853 